- Original Article

\title{
Analysis of the Comprehensiveness of Primary Care Clinics in Korea
}

\author{
Ha Jin Kim', Ji Yeh Shin'1, Yun Jun Yang ${ }^{2}$, Belong Cho' , Jae Moon Yun ${ }^{1, *}$ \\ 'Department of Family Medicine, Seoul National University Hospital, Seoul, Korea \\ ${ }^{2}$ Department of Family Medicine, Inje University Ilsan Paik Hospital, Goyang, Korea
}

Background: In the Republic of Korea, which medical specialties should take the responsibility for primary care and what the role of primary care should be are still unclear. In this study, we focused on the comprehensiveness of primary care to identify related factors.

Methods: The National Health Insurance Service (NHIS)-National Sample Cohort is a population-based cohort, sampled in the 2002 NHIS database and followed up until 2015. We used data collected from January 2014 to December 2015, including 20,423,832 outpatient visits in 19,557 office-based clinics. The Korean government has designated 52 simple or minor disease groups (SMDGs) to enhance the experience of patients who attend primary care for managing those diseases. We assessed comprehensiveness for each clinic as the number of SMDGs treated in each clinic for 2 years. We also identified the factors related to higher comprehensiveness, using logistic regression for analysis.

Results: The clinics included in the study had provided treatment for an average of 14 SMDGs during a 2-year period. Compared to general practitioners, internal medicine physicians presented higher comprehensiveness with an odds ratio (OR) of 2.29 (95\% confidence interval [CI], 2.03-2.59), and family medicine physicians illustrated higher comprehensiveness (OR, 4.96; 95\% CI, 3.59-6.83). Other specialties showed lower comprehensiveness than general practitioners. Clinics located in the capital city and metropolitan area tended to have lower comprehensiveness. Clinics hiring more doctors and having hospitalization facility showed higher comprehensiveness.

Conclusion: General physician, internal medicine, and family medicine are the fields providing comprehensive medical care in Korea. Clinics located in metropolitan area and capital city show lower comprehensiveness. The number of physicians is related to higher comprehensiveness of clinics.

Keywords: Primary Health Care; Comprehensiveness; Primary Care Clinic

Received: July 27, 2019, Revised: November 11, 2019, Accepted: December 6, 2019

*Corresponding Author: Jae Moon Yun https://orcid.org/0000-0003-0927-4721

Tel: +82-2-2072-2195, Fax: +82-2-766-3276, E-mail: jaemoon2@gmail.com 


\section{INTRODUCTION}

There are four key parts of primary care: first contact, continuity, comprehensiveness, and coordination. ${ }^{1)}$ As the burden of diseases per patient increases in an aging population, ${ }^{2,3)}$ comprehensive primary care has become more important. Comprehensive care means that all the medical aspects of one patient are dealt with by their primary care physician, ${ }^{4)}$ A more comprehensive primary care enables lower medical costs, less admission rates, and better patient satisfaction via greater efficiency. ${ }^{5)}$ Without comprehensive primary care, patients would experience fragmented care across numerous specialists. In Korea, which has lower medical costs and a surplus of specialists, fragmented care has long been a threat for primary care.

To improve the comprehensiveness aspect of primary care, it must be well defined and analyzed. Until now, studies on comprehensiveness were based on surveys of patients and clinicians. ${ }^{6-9)}$ Most surveys are focused on the scope of service provided; others attempted to measure whether acute or chronic symptoms are managed. ${ }^{10)} \mathrm{Al}-$ though these studies shed light on important factors for assessing comprehensiveness, they have several limitations: collected data are based on relatively small cohorts; results are subjected to individuals' experience and therefore cannot be standardized.

Recently, some studies have measured the comprehensiveness of primary care among physicians in different specialties using claim data from the national health system. ${ }^{7,11)}$ A few studies utilized the International Classification of Diseases (ICD) codes or diagnostic clusters (120 groups of diagnostic codes with similar pathophysiology and similar treatment) to measure the conditions treated. However, these methods cannot include the importance of each disease in primary care environment; moreover, there was no set standard based on which they could evaluate comprehensiveness within the practice of one primary care physician.

This study aims to define and standardize the comprehensiveness of primary care through statistical analysis of data extracted from the National Health Insurance Service (NHIS) that focused on the diseases commonly occurring in a primary care environment. Furthermore, we hope to describe and assess the factors of comprehensiveness with qualitative indices from our analysis.

\section{METHODS}

\section{Data Source and Study Population}

The NHIS National Sample Cohort is a population-based cohort, which was sampled in the 2002 NHIS database and followed up until 2015 . NHIS enrollees cover approximately $97 \%$ of all Koreans, and this cohort includes 1,000,000 individuals-approximately a $2 \%$ random sample of NHIS enrollees. We used data collected from January 2014 to December 2015, including 22,275,040 outpatient attendances at 29,912 office-based clinics.

From this pool, we excluded the clinics where the number of outpatient attendees was less than 400 over a 2-year period, to eliminate outliers. Given that the database is based on $2 \%$ random sampling, 400 visits can be interpreted as approximately 20,000 visits before sampling. This translates to approximately $30-40$ patients per each business day, which is much less than the average number of patients in the primary care environment in Korea. We finally included 19,557 primary care clinics and 20,423,832 outpatient attendances (Figure 1).

The Korean government has created a list of common diseases to encourage people to attend primary care clinics if they have those specific medical problems. If patients attend general hospitals with these medical problems, they would need to pay higher charges for their medication. The list comprises 52 groups of diseases, which are termed 'simple or minor disease groups' (SMDGs). The classification of diagnosis is based on the ICD-10 criteria.

\section{Measures}

\section{1) Comprehensiveness}

We calculated the number of SMDGs dealt with at each primary care clinic at least once over a 2-year period based on the diagnosis records. We assessed the comprehensiveness with this number. The more SMDGs they manage, the more the clinics are considered to provide comprehensive primary care. We categorized all the clinics into four groups based on the number of SMDGs each clinic manages, with cutoff values of lower quartile (25th percentile), median, and higher quartile (75th percentile). Because there are no defined measures for comprehensiveness, we used this score and tried to utilize the 25th percentile, median, and 75th percentile as the cutoff values.

\section{2) Clinic factors}

Clinic information included the number of physicians and nurses, the presence of a facility for hospitalization, geographical location, and specialty of each physician. The information for each clinic was used based on the data from 2014, so possible movement or closure of the clinic was not considered in the analysis. Specialty was defined as the single medical specialty the clinic registered to the administrative authority, even if the clinic hired several medical doctors who had different specialties. Only specialties responsible for more than $2 \%$ of primary care were included in the study. In Korea, general practitioner refers to a physician who has not received residency training.

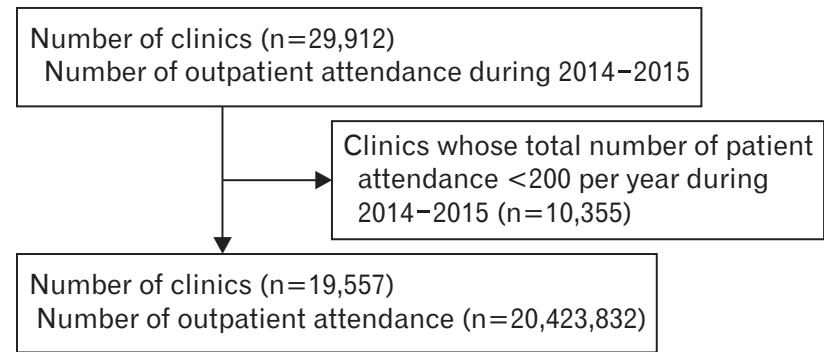

Figure 1. Flow chart showing selection of subjects for the study. 


\section{3) Most common SMDGs}

We ranked the most common 10 SMDGs among 52 SMDGs to identify which SMDGs were most common in outpatient-based clinics. We also measured which specialties are engaged in treating those diseases. Specialties that were responsible for managing more than $1 \%$ patients of each SMDG are expressed on the figure.

\section{Statistical Analysis}

We employed the chi-square test to discern differences among comprehensiveness groups for each characteristic. We used multivariable logistic regression to discern the elements that affected greater continuity. We divided the subjects into two groups, the higher comprehensiveness group and lower comprehensiveness group, using the median value as the cutoff. That is, we defined the higher comprehensiveness group as clinics that provide treatment for greater than or equal to 14 SMDGs and the lower one as clinics that provide treatment for lesser than 14 SMDGs. Then, we performed regressions with all the variables from the baseline characteristics included in the analysis. Multi- variate analyses were performed with adjustments for specialty of physician, number of physicians and registered nurses, and facility for hospitalization. Only specialties responsible for more than 3\% of primary care were included in the analysis. Odds ratios (ORs) and their 95\% confidence intervals (CI) were measured. All analyses were performed with the SAS Enterprise Guide ver. 7.1 (SAS Institute Inc., Cary, NC, USA). Two-sided P-values $<0.05$ were considered statistically significant.

\section{RESULTS}

\section{Characteristics of Clinics}

A total of 19,557 primary care clinics were included in the study. Of the study population, most of the clinic physicians were general practitioners (4,514 clinics, $23.1 \%$ ), followed by internal medicine physicians (3,686 clinics, $18.8 \%)$ and otolaryngologists $(2,050,10.5 \%)$. Among the clinics studied, $20.8 \%$ were located in Seoul, the capital city, and $21.8 \%$ were located in the Gyeonggi province. Most clinics had a single physi-

Table 1. Characteristics of clinics

\begin{tabular}{|c|c|c|c|c|c|c|}
\hline \multirow{2}{*}{ Characteristic } & \multirow{2}{*}{ Overall } & \multicolumn{4}{|c|}{ No. of simple or minor disease groups treated at a clinic } & \multirow{2}{*}{ P-value } \\
\hline & & $<8$ & $8-13$ & $14-18$ & $>18$ & \\
\hline No. of clinics & $19,557(100.0)$ & $4,572(23.4)$ & $4,598(23.5)$ & $4,739(24.2)$ & $5,648(28.9)$ & \\
\hline Specialty* & & & & & & $<0.01$ \\
\hline General practitioner & $4,514(23.1)$ & $386(2.0)$ & $668(3.4)$ & $1,131(5.8)$ & 2,329 (11.9) & \\
\hline Internal medicine & $3,686(18.8)$ & $41(0.2)$ & $414(2.1)$ & $1,388(7.1)$ & $1,843(9.4)$ & \\
\hline General surgery & $471(2.4)$ & $57(0.3)$ & $81(0.4)$ & $125(0.6)$ & $208(1.1)$ & \\
\hline Orthopedic surgery & $1,778(9.1)$ & $245(1.3)$ & $1,005(5.1)$ & $339(1.7)$ & $189(1.0)$ & \\
\hline Anesthesiology & $517(2.6)$ & $272(1.4)$ & $180(0.9)$ & $48(0.2)$ & $17(0.1)$ & \\
\hline Obstetrics and gynecology & $498(2.5)$ & $376(1.9)$ & $40(0.2)$ & $47(0.2)$ & $35(0.2)$ & \\
\hline Pediatrics & $1,890(9.7)$ & $17(0.1)$ & $494(2.5)$ & $969(5.0)$ & $410(2.1)$ & \\
\hline Ophthalmology & $1,239(6.3)$ & $1,209(6.2)$ & $30(0.2)$ & 0 & 0 & \\
\hline Otorhinolaryngology & $2,050(10.5)$ & $358(1.8)$ & $1,219(6.2)$ & $413(2.1)$ & $60(0.3)$ & \\
\hline Dermatology & $686(3.5)$ & $668(3.4)$ & $17(0.1)$ & 0 & $1(0.0)$ & \\
\hline Urology & $562(2.9)$ & $455(2.3)$ & $101(0.5)$ & $6(0.0)$ & 0 & \\
\hline Family medicine & 648 (3.3) & 0 & $43(0.2)$ & $151(0.8)$ & $454(2.3)$ & \\
\hline Others & $1,018(5.2)$ & $488(2.5)$ & $708(3.6)$ & $122(0.6)$ & $102(0.5)$ & \\
\hline Location & & & & & & $<0.01$ \\
\hline Seoul (capital city) & $4,065(20.8)$ & $1,055(5.4)$ & $1,002(5.1)$ & $982(5.0)$ & $1,026(5.2)$ & \\
\hline Gyeonggi (province) & $4,268(21.8)$ & $933(4.8)$ & $956(4.9)$ & $1,055(5.4)$ & $1,325(6.8)$ & \\
\hline Metropolitan city & $5,176(26.5)$ & $1,298(6.6)$ & $1,255(6.4)$ & $1,226(6.3)$ & $1,393(7.1)$ & \\
\hline Others & $5,979(30.6)$ & $1,276(6.5)$ & $1,366(7.0)$ & $1,461(7.5)$ & $1,879(9.6)$ & \\
\hline No. of physicians & & & & & & $<0.01$ \\
\hline 1 & $16,187(82.8)$ & $3,672(18.8)$ & 4,086 (20.9) & $4,116(21.0)$ & $4,313(22.1)$ & \\
\hline 2 & 2,322 (11.9) & $609(3.1)$ & $377(1.9)$ & $450(2.3)$ & $886(4.5)$ & \\
\hline$\geq 3$ & $1,048(5.4)$ & $291(1.5)$ & $135(0.7)$ & $173(0.9)$ & $449(2.3)$ & \\
\hline No. of registered nurses & & & & & & $<0.01$ \\
\hline 0 & $14,618(74.7)$ & $3,469(17.7)$ & $3,514(18.0)$ & $3,584(18.3)$ & $4,051(20.7)$ & \\
\hline 1 & 3,255 (16.6) & $688(3.5)$ & $749(3.8)$ & $781(4.0)$ & $1,037(5.3)$ & \\
\hline$\geq 2$ & $1,684(8.6)$ & $415(2.1)$ & $335(1.7)$ & $374(1.9)$ & $560(2.9)$ & \\
\hline Hospitalization facility & & & & & & $<0.01$ \\
\hline No & $14,992(76.7)$ & $3,546(18.1)$ & 3,092 (15.8) & $3,788(19.4)$ & $4,566(23.3)$ & \\
\hline Yes & 4,565 (23.3) & $1,026(5.2)$ & $1,506(7.7)$ & $951(4.9)$ & $1,082(5.5)$ & \\
\hline
\end{tabular}

Values are presented as number (\%).

*Only specialties responsible for more than $2 \%$ of primary care were included. Specialties with lesser than $2 \%$ are included in 'others'. 
cian $(82.8 \%)$ and no registered nurses (74.7\%). Most of the clinics (76.7\%) did not have facilities for hospitalization.

We measured comprehensiveness as the number of SMDGs each clinic provided treatment for at least once over the 2-year period. Among 52 SMDGs, clinics provided treatment for an average of 14 SMDGs (standard deviation=7.3), which is the same as the median value, with eight SMDGs for the 25th percentile and 19 SMDGs for the 75 th percentile. We categorized the clinics into four groups according to the level of comprehensiveness using the cutoff values of 25th percentile, median, and 75th percentile. Group 1, which serves from 0 to 7 SMDGs; group 2, from 8 to 13 SMDGs; group 3, from 14 to 18; and group 4, more than 18 SMDGs.

Specialty of the physician, location of the clinic, number of physicians and registered nurses, and presence of hospitalization facility showed significant differences in distributions among the four groups $(\mathrm{P}<0.05)$ (Table 1).

\section{Most Common Simple or Minor Disease Groups}

We identified how many times each SMDGs were treated over the 2 -year period. The 10 most common SMDGs were acute bronchitis, hypertension, allergic rhinitis, diabetes mellitus, acute tonsillitis, upper respiratory infection, acute sinusitis, acute pharyngitis, allergic contact dermatitis, and gastritis (Supplementary Table 1). Diseases related to respiratory infection, including acute bronchitis, acute tonsillitis, upper respiratory infection, acute sinusitis, and acute pharyngitis, were usually managed by general practitioners, internal medicine physicians, pediatricians, and otolaryngologists. Chronic diseases such as hypertension and diabetes mellitus were primarily managed by internal medicine physicians (more than $50 \%$ ), followed by general practitioners and family medicine physicians. General practitioners and internal medicine physicians were found to be engaged in all 10 of the most common SMDGs; family medicine physicians, otolaryngologists, and pediatricians were found to be engaged in most of the 10 commonest SMDGs (Figure 2).

\section{Factors Related to Comprehensiveness}

We divided all the clinics into two groups: the higher comprehensive group and lower comprehensive group. We figured out which factors of the clinics were related to higher comprehensiveness. Compared to general practitioners, internal medicine physicians presented higher comprehensiveness, with an OR of 2.29 (95\% CI, 2.03-2.59), and family medicine physicians illustrated higher comprehensiveness (OR, 4.96; 95\% CI, 3.59-6.83). Other specialties showed lower comprehensiveness than general practitioners. Clinics located in the capital city and metropolitan area were more likely to show lower comprehensiveness, but this difference was not statistically significant. The clinics with more doctors and hospitalization facility showed higher comprehensiveness. Regarding the number of registered nurses, clinics hiring a single nurse showed higher comprehensiveness, but clinics hiring two or more nurses showed significantly lower comprehensiveness (Table 2).

When we used the 1st quartile as the cutoff value, which is eight SMDGs, we found that pediatricians showed much higher comprehensiveness than general practitioners when compared to the result above. This was because this list of SMDGs comprised diseases that were common in adults, not in children; therefore, the number of SMDGs treated by pediatricians was automatically limited. Ophthalmologists and dermatologists are considered to provide highly specialized care, so they show very low comprehensiveness.

\section{DISCUSSION}

In our study, internal medicine and family medicine physicians were found to provide more comprehensive care than general practitioners. Our findings suggest that the comprehensiveness of primary care increases in proportion to the number of doctors in a clinic.

In Korea, general practitioners, internists, and family medicine physicians deal with a large number of diseases. Internal medicine physicians and family medicine physicians showed more comprehensiveness in their care compared to general practitioners. This is because the two specialties' areas encompass the majority of illnesses dealt

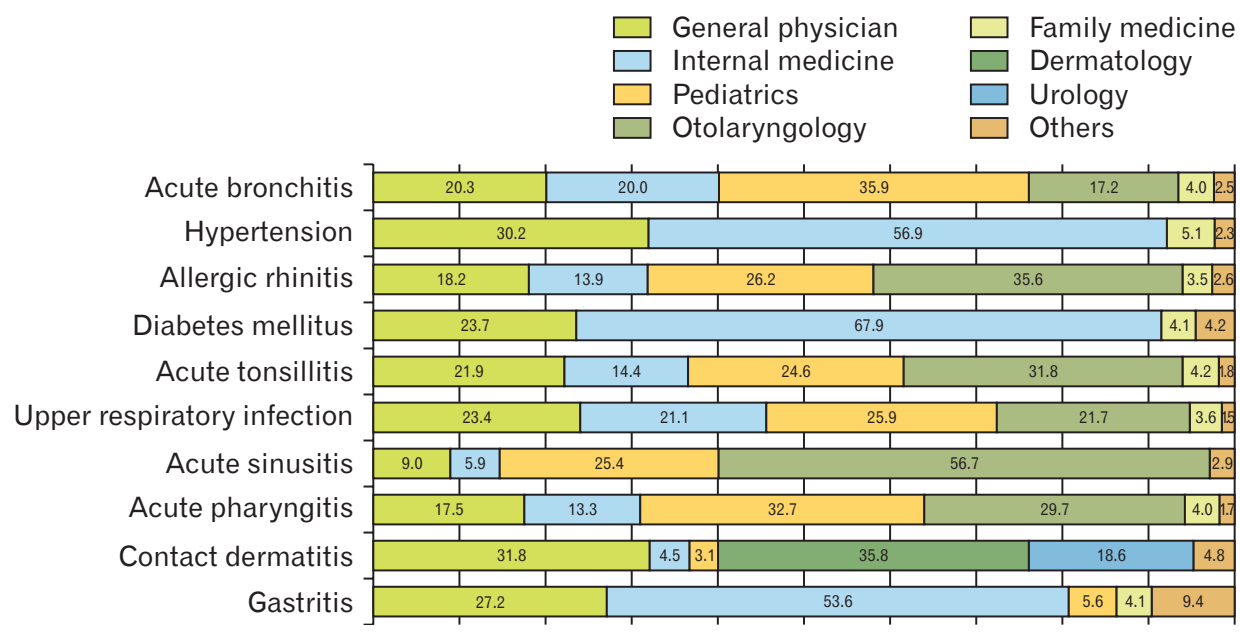

Figure 2. Ratio of the specialties of the clinic for treating most common 10 simple or minor disease groups. Values are presented as $\%$. 
Table 2. Factors related to higher comprehensiveness

\begin{tabular}{|c|c|}
\hline Variable & Odds ratio ( $95 \%$ confidence interval) \\
\hline \multicolumn{2}{|l|}{ Specialty $^{*}$} \\
\hline General practitioner & 1.00 (Reference) \\
\hline Internal medicine & $2.29(2.03-2.59)$ \\
\hline Orthopedic surgery & $0.10(0.09-0.12)$ \\
\hline Pediatrics & $0.82(0.72-0.93)$ \\
\hline Ophthalmology & Not available ${ }^{\dagger}$ \\
\hline Otorhinolaryngology & $0.08(0.07-0.09)$ \\
\hline Dermatology & $<0.01(<0.01-<0.01)$ \\
\hline Family medicine & $4.96(3.59-6.83)$ \\
\hline \multicolumn{2}{|l|}{ Location } \\
\hline Seoul (capital city) & 1.00 (Reference) \\
\hline Gyeonggi (province) & $0.93(0.83-1.04)$ \\
\hline Metropolitan city & $1.20(1.07-1.35)$ \\
\hline Others & $0.94(0.84-1.05)$ \\
\hline \multicolumn{2}{|l|}{ No. of physicians } \\
\hline 1 & 1.00 (Reference) \\
\hline 2 & $2.45(2.12-2.84)$ \\
\hline$\geq 3$ & $2.72(2.12-3.48)$ \\
\hline \multicolumn{2}{|l|}{ No. of registered nurses } \\
\hline 0 & 1.00 (Reference) \\
\hline 1 & $1.05(0.94-1.18)$ \\
\hline$\geq 2$ & $0.68(0.58-0.80)$ \\
\hline \multicolumn{2}{|l|}{ Hospitalization facility } \\
\hline No & 1.00 (Reference) \\
\hline Yes & $1.44(1.28-1.62)$ \\
\hline
\end{tabular}

*Only specialties responsible for more than $3 \%$ of primary care were included. ${ }^{\text {TTe }}$ number of observation is 0 , so calculation of odds ratio is impossible.

with in primary care. Furthermore, patients expect better care from specialists and therefore tend to rely on them for personal medical care. $^{12-14)}$

Our result showed lower comprehensiveness of primary care within the capital city (Seoul) and the metropolitan cities (administrative district of more than 1 million population); in comparison, higher comprehensiveness was seen in the Gyeonggi province. The reason may be the smaller density of clinic per person ratio in the Gyeonggi province compared to the density in the capital and metropolitan cities.

The number of physicians per clinic also contributed to the comprehensiveness of primary care. Each physician has different capabilities; ${ }^{15)}$ thus, hiring more physicians can enable the clinic to provide care for a greater variety of patients. Therefore, we suggest adopting group practice within a clinic to increase comprehensiveness. Studies from other countries already suggest that team practice leads to higher quality care, more accurate diagnosis, and increased satisfaction of patients in primary care settings. ${ }^{14,16,17)}$

A larger number of registered nurses per clinic is associated with lower comprehensiveness. However, in Korea, it is very common for assisting nurses to replace registered nurses at primary care clinics. Most clinics hire only assisting nurses, not registered nurses. According to our results, almost $75 \%$ of clinics do not hire registered nurses. Although the data does not include the assisting nurses' employment status, a considerable number of those clinics are assumed to hire as- sisting nurses based on primary care circumstances. Since the number of registered nurses does not reflect the accurate status of clinic personnel, it is difficult to comprehensively understand their relationship. Further research on this topic is required by collecting sufficient employment-related data.

This study defines the comprehensiveness of primary care using data selected from the NHIS. To the best of our knowledge, this is the first study to assess different factors related to comprehensiveness using statistical analysis. ${ }^{6,7,9)}$

However, there are limitations to this study. The SMDGs used in this study were chosen and adapted to suit the Korean health care scenario, and may not be consistent with the groups of diseases that need to be treated in primary care settings elsewhere. ${ }^{18,19)}$ For example, the lower comprehensiveness of pediatrics was because only a few of the SMDG diseases in this study were relevant to pediatric patients. Therefore, it is important to first establish the group of diseases seen in primary care settings; then, the comprehensiveness of primary care settings can be defined based on the established diseases.

Lastly, our study is based on diagnosis-related data extracted from Korean claim data, and thus has yet to be implemented abroad. Because claim data are heavily influenced by insurance standards, differing insurance standards in various medical systems may reveal entirely different results. This will need further studies.

In conclusion, although a variety of specialties provide outpatient services in Korea, general physicians, internal medicine physicians, and family medicine physicians were found to provide comprehensive medical care; moreover, clinics located in metropolitan areas and the capital city have lower comprehensiveness. The number of physicians is related to the higher comprehensiveness of clinics.

\section{CONFLICT OF INTEREST}

No potential conflict of interest relevant to this article was reported.

\section{ACKNOWLEDGMENTS}

We declare that that we are funded by Korean Medical Association with grant number 2018-09.

\section{SUPPLEMENTARY MATERIALS}

Supplementary materials can be found via https://doi.org/10.4082/ kjfm.19.0120. Supplementary Table 1. Most common 10 SMDGs and the number of diagnosis record during 2 years.

\section{ORCID}

Ha Jin Kim: https://orcid.org/0000-0002-7632-0882

Ji Yeh Shin: https://orcid.org/0000-0003-4736-1316

Yun Jun Yang: https://orcid.org/0000-0003-3428-1587

Belong Cho: https://orcid.org/0000-0001-9558-689X 
Jae Moon Yun: https://orcid.org/0000-0003-0927-4721

\section{REFERENCES}

1. Bodenheimer T, Ghorob A, Willard-Grace R, Grumbach K. The 10 building blocks of high-performing primary care. Ann Fam Med 2014; 12:166-71.

2. Dall TM, Gallo PD, Chakrabarti R, West T, Semilla AP, Storm MV. An aging population and growing disease burden will require a large and specialized health care workforce by 2025. Health Aff (Millwood) 2013;32:2013-20.

3. Boyle JP, Thompson TJ, Gregg EW, Barker LE, Williamson DF. Projection of the year 2050 burden of diabetes in the US adult population: dynamic modeling of incidence, mortality, and prediabetes prevalence. Popul Health Metr 2010;8:29.

4. Starfield B, Shi L, Macinko J. Contribution of primary care to health systems and health. Milbank Q 2005;83:457-502.

5. Bazemore A, Petterson S, Peterson LE, Phillips RL Jr. More comprehensive care among family physicians is associated with lower costs and fewer hospitalizations. Ann Fam Med 2015;13:206-13.

6. Schoen C, Osborn R, Doty MM, Squires D, Peugh J, Applebaum S. A survey of primary care physicians in eleven countries, 2009: perspectives on care, costs, and experiences. Health Aff (Millwood) 2009;28: w1171-83.

7. O'Malley AS, Rich EC. Measuring comprehensiveness of primary care: challenges and opportunities. J Gen Intern Med 2015;30 Suppl 3(Suppl 3):S568-75.

8. Franks P, Clancy CM, Nutting PA. Defining primary care: empirical analysis of the National Ambulatory Medical Care Survey. Med Care 1997;35:655-68.

9. Engels Y, Campbell S, Dautzenberg M, van den Hombergh P, Brinkmann H, Szecsenyi J, et al. Developing a framework of, and quality indicators for, general practice management in Europe. Fam Pract 2005;
22:215-22.

10. DesRoches C, Rich E. Collecting data on physicians and their practices: final report to AHRQ. Washington (DC): Mathematica Policy Research; 2014.

11. Rosenblatt RA, Hart LG, Baldwin LM, Chan L, Schneeweiss R. The generalist role of specialty physicians: is there a hidden system of primary care? JAMA 1998;279:1364-70.

12. Kraska RA, Weigand M, Geraedts M. Associations between hospital characteristics and patient satisfaction in Germany. Health Expect 2017;20:593-600.

13. Ruiz-Moral R, Perula de Torres LA, Jaramillo-Martin I. The effect of patients' met expectations on consultation outcomes: a study with family medicine residents: version 2. J Gen Intern Med 2007;22:86-91.

14. Chang CS, Chen SY, Lan YT. Service quality, trust, and patient satisfaction in interpersonal-based medical service encounters. BMC Health Serv Res 2013;13:22.

15. Wilson A, Childs S. The relationship between consultation length, process and outcomes in general practice: a systematic review. Br J Gen Pract 2002;52:1012-20.

16. Bower P, Campbell S, Bojke C, Sibbald B. Team structure, team climate and the quality of care in primary care: an observational study. Qual Saf Health Care 2003;12:273-9.

17. Friedberg MW, Coltin KL, Pearson SD, Kleinman KP, Zheng J, Singer JA, et al. Does affiliation of physician groups with one another produce higher quality primary care? J Gen Intern Med 2007;22:1385-92.

18. Macinko J, Starfield B, Shi L. The contribution of primary care systems to health outcomes within Organization for Economic Cooperation and Development (OECD) countries, 1970-1998. Health Serv Res 2003;38:831-65.

19. Marshall M, Klazinga N, Leatherman S, Hardy C, Bergmann E, Pisco L, et al. OECD Health Care Quality Indicator Project. The expert panel on primary care prevention and health promotion. Int J Qual Health Care 2006;18 Suppl 1:21-5. 